\title{
Eine neue Methode zur quantitativen Fluorbestimmung.
}

\author{
Von \\ Gunnar Stakck.
}

\begin{abstract}
Bei einer Untersuchung über ammoniakalische Verbindungen mit Fluor im Komplex machte sich bei mir das Bedürfnis einer
\end{abstract} schnellen Fluorbestimmungsmethode dringend geltend. Keine der geläufigen Methoden erfüllt nur annähernd die Forderung zugleich bequem und exakt zu sein. Die einzige Fällungsmethode, mit Calciumchlorid in alkalischer Lösung, ist schon von anderer Seite $^{1}$ mit Recht verworfen worden. Die Destillationsmethoden, obgleich bessere Resultate gebend, sind ziemlich zeitraubend, besonders wenn eine grölsere Anzahl Bestimmungen auszuführen sind.

Da es sich bei mir in erster Linie um lösliche Fluorverbindungen handelte, habe ich mich bemüht, eine neue Fällungsmethode zu finden. Ich fand auch bald ein Fluorid, das in bezug auf Schwerlöslichkeit, leichte Filtrierbarkeit usw. ziemlich hohen Anforderungen zu entsprechen schien. Dasselbe ist eine zuerst von Berzecrus ${ }^{2}$ dargestellte Verbindung zwischen Blei, Fluor und Chlor, $\mathrm{PbFCl}$, die man durch Fällen von Natriumfluorid mit überschüssiger Bleichloridlösung erhält. Später ist dieselbe von Fonzes-Dracos ${ }^{3}$ erwähnt worden, näher untersucht aber nicht.

Wird die neutrale Lösung eines Fluorides mit überschüssiger, nahe gesättigter Bleichloridlösung versetzt, so fällt obige Verbindung als weifser Niederschlag aus. Derselbe ist sehr schwer und setzt sich schnell ab. Die Zusammensetzung der bei $140-150^{\circ}$ getrockneten Substanz ist, durch Bestimmung des Chlors ermittelt,

$\begin{array}{lcc}0 & \text { Berechnet: } & \text { Gefunden: } \\ \mathrm{Pb} & 79.18 & - \\ \mathrm{F} & 7.26 & - \\ \mathrm{Cl} & 13.56 & 13.58 \quad 13.55\end{array}$

Die Fällung ist aufserordentlich leicht filtrierbar, ja, stellt eine in dieser Hinsicht vollkommen ideale Substanz dar. Die Brauch-

1 SeEManN, Zeitschr. analyt. Chem. 44 (1905), 343.

2 Lehrbuch der Chemie, 4. Aufl., Bd, 4, S. 497.

${ }^{3}$ Bull. soc. chim. [3] 17 (1897), 346-348.

Z. anorg. Chem. Bd. 70. 
barkeit für den angegebenen Zweck hängt also nur von der Löslichkeit in Wasser und anderen Solvenzien $a b$.

Die folgenden Angaben der Löslichlieit von $\mathrm{PbFCl}$ in Wasser, Bleichloridlösungen, Salzsäure und Essigsäure sind einer später zu veröffentlichenden, gröIseren Arbeit entnommen, worin die Verbindungen von Bleifluorid mit Bleichlorid, -bromid und -jodid ausführlich behandelt werden. ${ }^{1}$

Tabelie 1.

$100 \mathrm{~g}$ Wasser lösen

$\begin{array}{cccc}\text { bei } 0^{\circ} & 18^{\circ} & 25^{\circ} & 100^{\circ} \\ 0.0211 & 0.0325 & 0.0370 & 0.1081 \mathrm{~g} \mathrm{PbFCl} \\ 0.0807 & 0.1241 & 0.1415 & \text { Millimole } \mathrm{PbFCl}\end{array}$

Tabelle 2.

Löslichkeit iu Bleichloridlösungen, Äq. Normal. = N.

\begin{tabular}{|c|c|c|c|c|}
\hline Temp. in ${ }^{\circ}$ & $\mathrm{N}$ & $100 \mathrm{cer}$ & lösen & Bemcrkung: \\
\hline 18 & 0.0100 & $0.0020 \mathrm{~g}$ & $\mathrm{PbFCl}$ & \\
\hline 18 & 0.0195 & 0.0016 & " & Als Dirnerenz von zönen \\
\hline 18 & 0.0495 & 0.0002 & $"$ & dieseZiffern keine grofse \\
\hline 25 & 0.00996 & 0.0030 & ", & Genanigkeit bean- \\
\hline 25 & 0.0195 & 0.0008 & $"$ & $\begin{array}{l}\text { spruchen } \\
\text { sprestat }\end{array}$ \\
\hline 25 & 0.0392 & 0.0005 & $\because$ & \\
\hline
\end{tabular}

Tabelle 3.

Löslichkeit in Salzsäure und in Essigsäure, Äq.-Normal. $=\mathbf{N}$, bei $25^{\circ}$.

$\mathrm{N} \quad 100 \mathrm{ccm}$ lösen

\begin{tabular}{|c|c|c|c|c|c|c|c|c|}
\hline $\mathrm{HCl}$ & 0.0535 & $0.0758 \mathrm{~g}$ & l'bFCl & aus & gefund. & $0.0817 \mathrm{~g}$ & $\mathrm{PbCl}_{2}$ & be \\
\hline & 0.1069 & 0.1006 & ," & "r & $n$ & 0.1085 &, & ; \\
\hline $\mathrm{CH}_{3} \mathrm{CO}_{2} \mathrm{H}$ & 0.0518 & 0.0512 & " & & & & & \\
\hline & 0.1055 & 0.0561 & 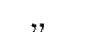 & & & & & \\
\hline
\end{tabular}

Die aus der Essigsäurelösung ausgeschiedene Trockensubstanz ist $\mathrm{PbFCl}$, wie durch Abrauchen mit $\mathrm{HCl}$ bewiesen wurde, wobei 0.0550 (bzw. $0.0605 \mathrm{~g}$ ) $\mathrm{PbCl}_{2}$ statt o.552 (bzw. 0.0605) $\mathrm{g} \mathrm{PbCl}_{2}$ erhalten wurden.

Aus den Tabellen wird ersichtlich, dafs die Löslichkeit in Wasser nicht unbeträchtlich ist; durch kleine Mengen von Salzoder Essigsäure wird dieselbe noch mehr erhöht; in Bleichlorid-

1 Es sind Abkühlungskurven der geschmolzenen Mischungen von $\mathrm{PbF}_{2}$ mit $\mathrm{PbCl}_{2}$, bzw. $\mathrm{PbBr}_{2}$ und $\mathrm{PbJ}_{2}$, aufgenommen, Löslichkeiten und Dissoziationsverhältnisse untersucht usw. 
lösungen, selbst ganz verdünnte, ist die Substanz dagegen praktisch unlöslich. Bei der Ausfällung von Fluor nach dieser Methode muls deshalb ein grofser Überschul's an fast gesättigter Bleichloridlösung zur neutralen Lösung gesetzt werden, beim Filtrieren muls Bleichloridlösung zum Aufspülen des Niederschlages angewandt und nur zuallerletzt reines Wasser zur Verdrängung des Bleichlorids benutzt werden. Nach diesem Prinzip sind eine grofse Anzahl Bestimmungen von Fluor in Fluornatriumlösungen mit durchgehendem Erfolg ausgeführt worden.

Im einzelnen wurde dabei folgendermafsen gearbeitet. Eine abgemessene Lösung von Natriumfluorid, die zwecks der Aufbewahrung in Glasgefälsen etwas alkalisch gemacht worden war, wurde mit verdünnter (etwa $1 / 10$-norm.) $\mathrm{HCl}$ vorsichtig neutralisiert (Phenolphtalein) und dann in der Kälte mit einem grolsen Überschufs von bei Zimmertemperatur gesättigter Bleichloridlösung versetzt. Für $50 \mathrm{ccm}$ Lösung, enthaltend ca. $0.1 \mathrm{~g} \mathrm{NaF}$, wurden dabei etwa $200 \mathrm{ccm}$ Bleilösung verwendet, für andere Mengen entsprechend weniger oder mehr. Wegen der Hydrolyse des Bleichlorids schlägt die Reaktion dabei in schwach sauer um, was ohne Belang ist. Nach einigem Stehen (gewöhnlich über Nacht) wird abfiltriert. Zum Aufnehmen der Fällung habe ich Neubauertiegel aus Platin oder Porzellan, ${ }^{1}$ gewöhnliche Goochtiegel mit Asbestfilter oder endlich Filtrierröhrchen aus Normalglas mit Glaswolle-A sbestpackung, alle gleich gut, benutzt. ${ }^{2}$ Der gesamte Niederschlag wird mit nicht ganz gesättigter Bleichloridlösung aufs Filter gebracht, dann einigemal mit Bleichloridlösung gewaschen, endlich das Bleichlorid durch 3-4 maliges Waschen durch reines Wasser verdrängt. Der Niederschlag wird bei 140 bis $150^{\circ} 2$ Stunden getrocknet und ist dann rein.

Wie gesagt, sind eine grofse Anzahl, etwa 200, Fluorbestimmungen nach dieser Methode ausgeführt worden. Mit wenigen Ausnahmen sind dieselben richtig ausgefallen. Als Beleg mögen nur folgende, von mir ausgeführten Bestimmungen angeführt werden, die in je $25 \mathrm{ccm}$ einer von meinem Assistenten durch Einwägen reinsten Fluornatriums dargestellten Lösung - mir von vornherein unbekannt - vorgenommen wurden.

${ }^{1}$ De VRIEs, Chem. Centrbl. 1909 II, 2058.

${ }^{2}$ Nach jeder Bestimmung muls der Niedersehlag aus dem Tiegel entfernt werden. Der grölste Teil kann leicht herausgeklopft werden, der Rest wird mit heifsem Wasser + etwas Salzsäure gelöst. 
Tabelle 4.

\begin{tabular}{c|c|c|c||c|c|c||c|c|c}
\hline \hline \multicolumn{2}{c|}{ PbFCl } & \multicolumn{2}{c||}{ Diff. } & \multicolumn{3}{c||}{ Fluor } & \multicolumn{2}{c}{$\%$ F in NaF } \\
\hline gef. & ber. & g & $\%$ & gef. & ber. & Diff. & gef. & ber. & Diff. \\
\hline \hline 0.4268 & 0.4259 & +0.0009 & 0.21 & 0.0310 & 0.0309 & +0.0001 & 45.34 & 45.24 & +0.10 \\
0.4290 & & +0.0031 & 0.73 & 0.0312 & & +0.0003 & 45.58 & & +0.34 \\
0.4239 & & -0.0020 & 0.47 & 0.0308 & & -0.0001 & 45.04 & & -0.20 \\
0.4258 & & -0.0001 & 0.02 & 0.0309 & & 0.0000 & 45.24 & & \pm 0 \\
0.4230 & & -0.0029 & 0.68 & 0.0307 & & -0.0002 & 44.94 & & -0.30 \\
0.4269 & & +0.0010 & 0.23 & 0.0310 & & +0.0001 & 45.35 & & +0.11 \\
0.4302 & & +0.0043 & 1.00 & 0.0313 & & +0.0004 & 45.70 & & +0.46 \\
0.4279 & & +0.0020 & 0.47 & 0.0311 & & +0.0002 & 45.46 & & +0.22 \\
0.4289 & & +0.0030 & 0.71 & 0.0312 & & +0.0003 & 45.57 & & +0.33 \\
0.4301 & & +0.0042 & 0.99 & 0.0313 & & +0.0004 & 45.70 & & +0.46 \\
0.4243 & & -0.0016 & 0.38 & 0.0308 & & -0.0001 & 45.08 & & -0.16 \\
0.4261 & & +0.0002 & 0.05 & 0.0309 & & 0.0000 & 45.27 & & +0.03 \\
0.4242 & & -0.0017 & 0.40 & 0.0308 & & -0.0001 & 45.07 & & -0.17 \\
0.4268 & & +0.0009 & 0.21 & 0.0310 & & +0.0001 & 45.34 & & +0.10 \\
0.4240 & & +0.0019 & 0.45 & 0.0308 & & -0.0001 & 45.05 & & -0.19 \\
0.4234 & & -0.0025 & 0.59 & 0.0308 & & -0.0001 & 44.99 & & -0.25 \\
0.4236 & & -0.0023 & 0.54 & 0.0308 & & -0.0001 & 45.01 & & -0.23 \\
& & & & & & & & &
\end{tabular}

Die Übereinstimmung ist befriedigend. Es unterliegt keinem $Z$ weifel, dals sich dieselbe noch weiter treiben läfst, wie tatsächlich in anderen von uns ausgeführten Serien der Fall war. Da der Niederschlag 14 mal schwerer als die gesuchte Fluormenge ist, so darf letztere nicht sehr grols genommen werden (höchstens $7 \mathrm{cg}$ ); andererseits werden auch beträchtliche, absolute Gewichtsdifferenzen im Niederschlag bei Umrechnung auf Fluor kolossal herabgesetzt.

Anstatt den Niederschlag als solchen zu wägen, kann man ihn auflösen und das darin enthaltene Chlor gravimetrisch oder titrimetrisch (nach VoLhard) bestimmen. Über Versuche mit einer gravimetrischen Chlorbestimmung berichtet folgende Tabelle. Der Niederschlag, auf Papierfilter genommen und mit kaltem Wasser vorsichtig gewaschen, wurde mit heifsem Wasser, unter Mitwirkung von einigen Kubikzentimeter verdünnter Salpetersäure in ein Becherglas gespült und $\mathrm{AgCl}$ in gewöhnlicher Weise ausgefällt.

Endlich kann man mit einer bekannten Bleichloridmenge fällen, zum bestimmten Volum verdünnen und nicht ausgefälltes Chlor in einem aliquoten Teil der Lösung bestimmen. Diesbezügliche Versuche sind gemacht; die Fehlerquellen sind aber dabei ziemlich grols, und die Resultate können sich den oben angeführten nicht 
Tabelle 5 .

\begin{tabular}{r|c|c}
\hline \hline \multicolumn{3}{|c}{ AgCl } \\
\hline gef. & ber. & \multicolumn{1}{c}{ Diff. } \\
\hline \hline 0.0714 & 0.0714 & 0.0000 \\
719 & & +0.0005 \\
716 & & +0.0002 \\
717 & & +0.0003 \\
704 & & -0.0010 \\
0.1794 & 0.1785 & +0.0009 \\
1796 & & +0.0011 \\
1793 & & +0.0008 \\
1796 & & +0.0011 \\
0.3544 & 0.3570 & -0.0026 \\
3557 & & -0.0013 \\
3554 & & -0.0016 \\
3564 & & -0.0006
\end{tabular}

an die Seite stellen. Diese Art der Ausführung könnte indessen von Bedeutung sein in Fällen, wo neben Fluor Anionen anwesend sind, die auch Blei ausfällen. Diese Aufgabe ist im Angriff genommen.

Der weitere Ausbau der Methode, die Übertragung derselben auf die praktische Fluorbestimmung in Mineralien, die Trennung des Fluors von anderen Anionen, insbesondere von Kieselsäure, die Analyse von Flufsspat usw. werden dem Verfasser vorbehalten.

Den Herren Studierenden E. GRUbB und E. Ericsson, die mir bei der Untersuchung behilflich waren, spreche ich meinen wärmsten Dank aus.

Stockholm, Analylisches Laboratorium der Hochschule.

Bei der Redaktion eingegangen am 1. Dezember 1910. 